\title{
Acute Zonal Occult Outer Retinopathy (AZOOR): a case report of vision improvement after intravitreal injection of Ozurdex
}

\author{
Yi Chun Kuo', Nancy Chen ${ }^{1}$ and Rong Kung Tsai ${ }^{2,3^{*}}$
}

\begin{abstract}
Background: AZOOR was first described by Gass in 1993 as a syndrome with rapid loss of one or more extensive zones of the outer retinal segments. It is characterized by photopsia, minimal funduscopic changes, and electroretinographic abnormalities. The efficacy of systemic steroids in treating AZOOR has been previously described and advocated by the concept of autoimmune retinopathy. However, the use of intravitreal of sustained-released steroid had not been mentioned to date.

Case presentation: A 34-year-old man had sudden onset of central scotoma and photopsia in the left eye. His visual acuity continued deteriorating. The visual field defect demonstrated bilateral enlarged blind spots and altitudinal defects. Fluorescein angiography (FA) showed nonspecific retinal inflammation, and an electroretinogram (ERG) illustrated decreased amplitude of the b wave in both eyes. Optical coherence tomography (OCT) examinations revealed parafoveal loss of the photoreceptor inner/outer segment (IS/OS) junction. Therefore, acute zonal occult outer retinopathy (AZOOR) was diagnosed. Although his vision did not improve under the initial treatment of systemic corticosteroid and calcium channel blocker, remarkable improvement was noticed after the intravitreal injection(IVI) of Ozurdex, consistent with the recovered IS/OS junction disruption.

Conclusions: We herein report a typical case of AZOOR, suggesting that the intravitreal injection of steroid may benefit in certain patients.
\end{abstract}

Keywords: Acute zonal occult outer retinopathy, Optical coherence tomography, Electroretinogram, Sustainedreleased steroid

\section{Background}

AZOOR was first described by Gass in 1993 as a syndrome with rapid loss of one or more extensive zones of the outer retinal segments. It occurs predominantly in young women, characterized by photopsia, minimal funduscopic changes, and electroretinographic abnormalities affecting one or both eyes $[1,2]$. The most common visual field (VF) defects are enlarged blind spots and scotoma connecting to blind spots [3]. Retinal pigment epithelial (RPE) atrophy and retinal vessel narrowing

\footnotetext{
* Correspondence: tsai.rk@gmail.com

${ }^{2}$ Institute of Eye Research, Buddhist Tzu Chi General Hospital, Tzu Chi

University, 707, Sec. 3, Chung-Yang Rd., Hualien 970, Taiwan

${ }^{3}$ Institute of Medical Sciences, Tzu Chi University, Hualien, Taiwan

Full list of author information is available at the end of the article
}

have been observed in many cases as sequelae [4]. Optical coherence tomography (OCT) examinations demonstrated loss and irregularity of the IS/OS boundary in scotomatous areas $[5,6]$. Fundus autofluorescence may also reveal a markedly hyperautofluorescent delineating line around the zonal area of RPE atrophy $[7,8]$. The cone outer segment tip (COST) line has been recognized as a thin, highly reflective line located between the IS/OS junction and the RPE, and abnormalities of the COST line are observed in AZOOR $[9,10]$. The efficacy of systemic steroids in treating AZOOR has been previously described. Visual improvement was achieved in most reported cases $[11,12]$. Herein, we present a patient whose symptoms 
did not subside with oral steroid use; however, he experienced visual improvement after receiving intravitreal injection of a sustained-released steroid.

\section{Case presentation}

The 34-year-old man was in good health until he failed to hit a ping-pong ball accurately one morning. Blackish dots appeared in the nasal upper visual field, and flashes blocked the vision in front of his left eye. He was myopic with -11.0 diopter (D) in the right eye (OD) and -15.0 in the left eye (OS), and he underwent LASIK surgery seven years ago. He visited our emergency department, where visual acuity (VA) revealed 20/20 (OD) and 20/32 (OS). No cells were noted at the anterior chamber (AC) or vitreous. His fundus seemed normal under dilated fundoscopy.

On the next day, vision decreased significantly to 20/25 (OD) and 20/63 (OS). Visual field (VF) examinations demonstrated bilateral enlarged blind spots and altitudinal defects (Fig. 1a-b). FA showed hyperfluorescence (OS) and inflammation of retinal small vessels (Fig. 2). OCT examinations revealed parafoveal loss of the photoreceptor IS/OS junction (Fig. 3a). ERG revealed decreased amplitude of the $\mathrm{b}$ wave in both eyes (Fig. 4a-b). According to his symptoms and examinations, AZOOR was diagnosed. We gave systemic corticosteroid (dexamethasone $6 \mathrm{mg}$ once a day) on the next day after day of onset. Nevertheless, his vision didn't improve after dexamethasone treatment for 9 days, so we tapered dexamethasone to $4 \mathrm{mg}$ and prescribed calcium channel blocker (amlodipine $5 \mathrm{mg}$ once a day). However, his VA did not improve for 3 weeks. Intravitreal injections (IVI) of Ozurdex (OS) was suggested and arranged. The next day after IVI of Ozurdex, his VA improved to 20/32 (OS). The course was smooth. Follow-up OCT showed improvement of the IS/OS junction disruption two weeks later (Fig. 3b). VA (OS) improved to 20/20, and the VF defect improved significantly (Fig.1c-d) as of one month after IVI. Owing to the appearance of the high myopia tilting disc and deep vertical enlarged cupping, the VF defects might be a summation of different components, AZOOR and myopia related glaucoma. Intraocular pressure (IOP) was elevated to $28 \mathrm{mmHg}$ initially after IVI; however, IOP remained
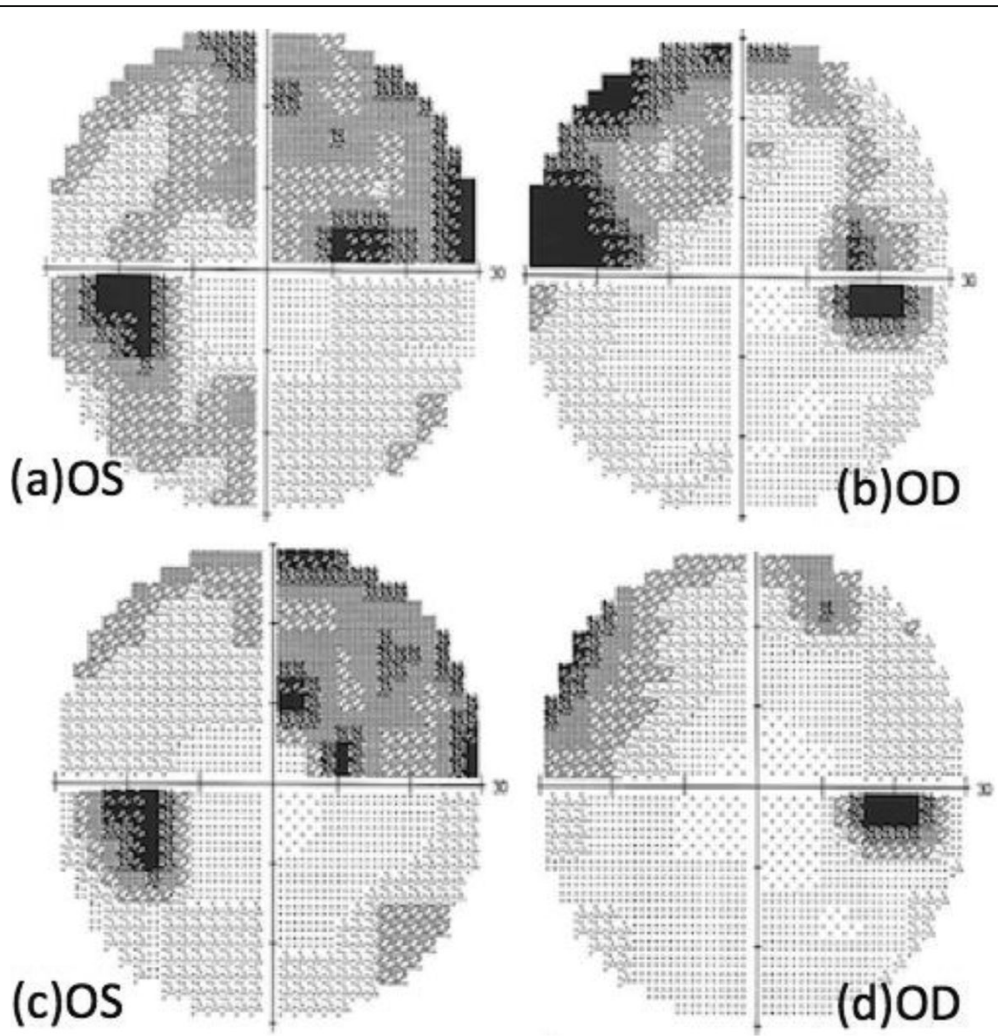

Fig. 1 VF defect (detected by Humphrey Visual Field Analyser 30-2) significantly subsided after one month of IVI of Ozurdex. (a, b) VF on the next day after onset of symptoms. (OD: Fixation losses: 1/21, false positive errors: $5 \%$ and false negative errors: $6 \%$. OS: Fixation losses: 0/21, false positive errors: $0 \%$ and false negative errors: $5 \%$ ) (c, d) One month after IVI, VF defect significantly subsided of both eyes. (OD: Fixation losses: 0/19, false positive errors: $4 \%$ and false negative errors: 7\%. OS: Fixation losses: 1/21, false positive errors: $0 \%$ and false negative errors: $1 \%)$ 


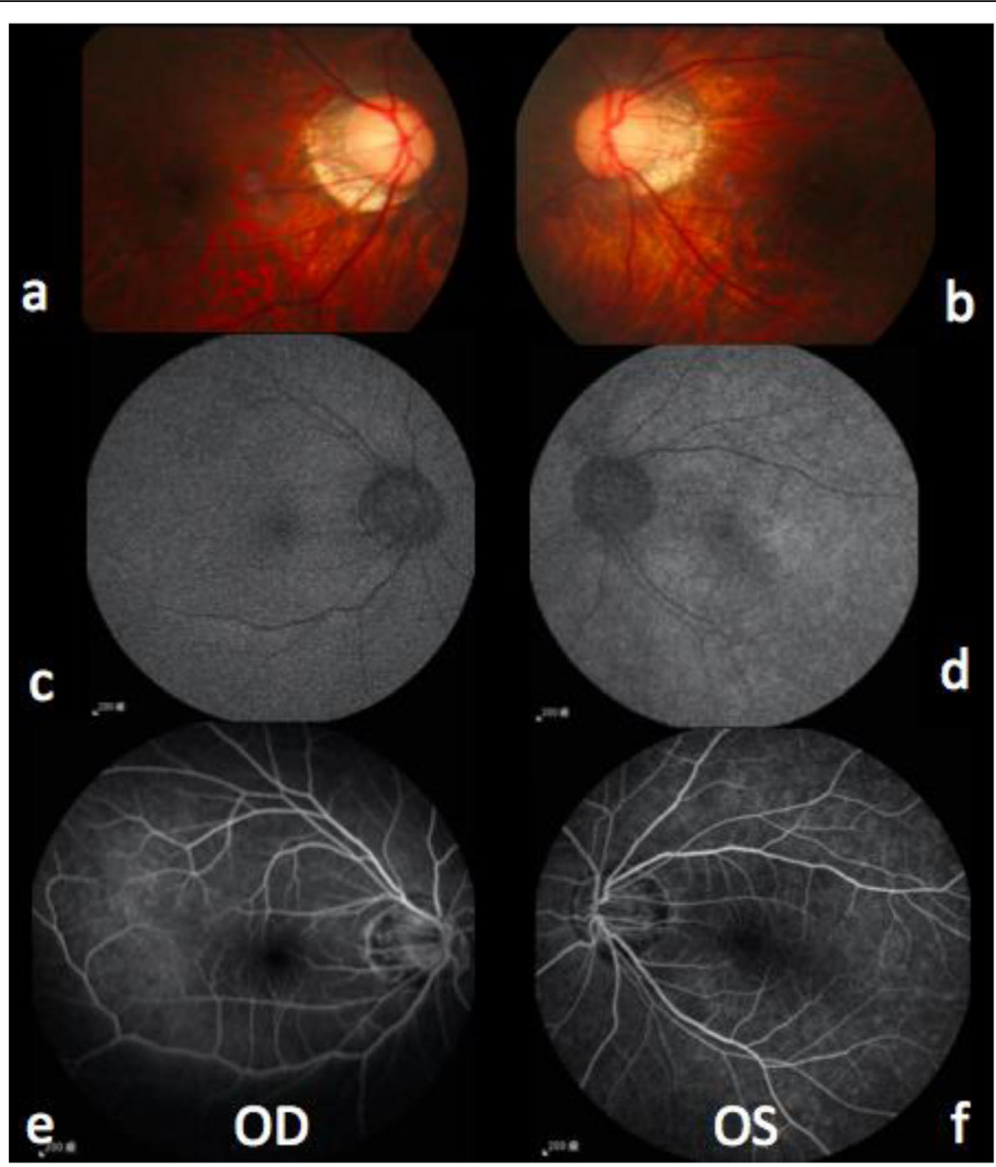

Fig. 2 Binocular images of color fundoscopy, fundus autofluorescence, and fluorescein angiography on the next day after onset of symptoms. Color fundoscopy (a-b) revealed no specific finding except myopic crescent. Compared to the right eye (c), fundus autofluorescence revealed hyper-autofluorescence of the left eye (d). Fluorescein angiography (e-f) revealed retina small vessels vasculitis inflammation of the left eye(f)

within normal range after 2 weeks of bimatoprost (Lumigan) treatment. At the recent follow-up after 13 months of the episode, his best corrected VA (OS) was 20/20. There was no cataract nor IOP elevation; meanwhile, OCT showed no sign of IS/OS junction disruption (Table 1).

\section{Discussion and conclusions}

The etiology of AZOOR remains controversial. An infectious viral process of the outer retina was suggested by Gass. [1] Autoimmune and inflammatory hypotheses were proposed by Jampol and Becker $[13,14]$. Other possible mechanisms includes fungal infiltration, [15, 16] polycythemia vera, [17] toxic retinopathy, $[14,18,19]$ and anti-retinal antibody $[20,21]$. Heckenlively and Ferreyra [22] reviewed retinal disorders evoked by autoantibodies and established a clinical term of autoimmune retinopathy (AIR). They stated that AIR could be a secondary complication of
AZOOR, implying that an autoimmune reaction mediated by humoral immunity plays an important role in the disease process of AZOOR. Logically, steroid therapy might be effective in some AZOOR patients $[11,23]$.

Various treatments have been attempted in patients with AZOOR, including systemic corticosteroids, [11, 12, 24] other systemic immunosuppressive agents, [4, 25] and antimicrobial agents, [19] but their effects were not conclusive yet. Based on the proposed mechanisms of anti-retinal antibody, [20-22] AZOOR could be an autoimmune disease with antiretinal antibody leakage from the disc margin with spread of immune substance under the retina. [21] Previous studies have showed effectiveness of systemic steroid. Saito [24] gave fourteen patients intravenous methylprednisolone $1000 \mathrm{mg} /$ day for 3 days, oral prednisolone $30 \mathrm{mg} /$ day for 7 days, intravenous methylprednisolone $1000 \mathrm{mg} /$ day for 3 days, with 


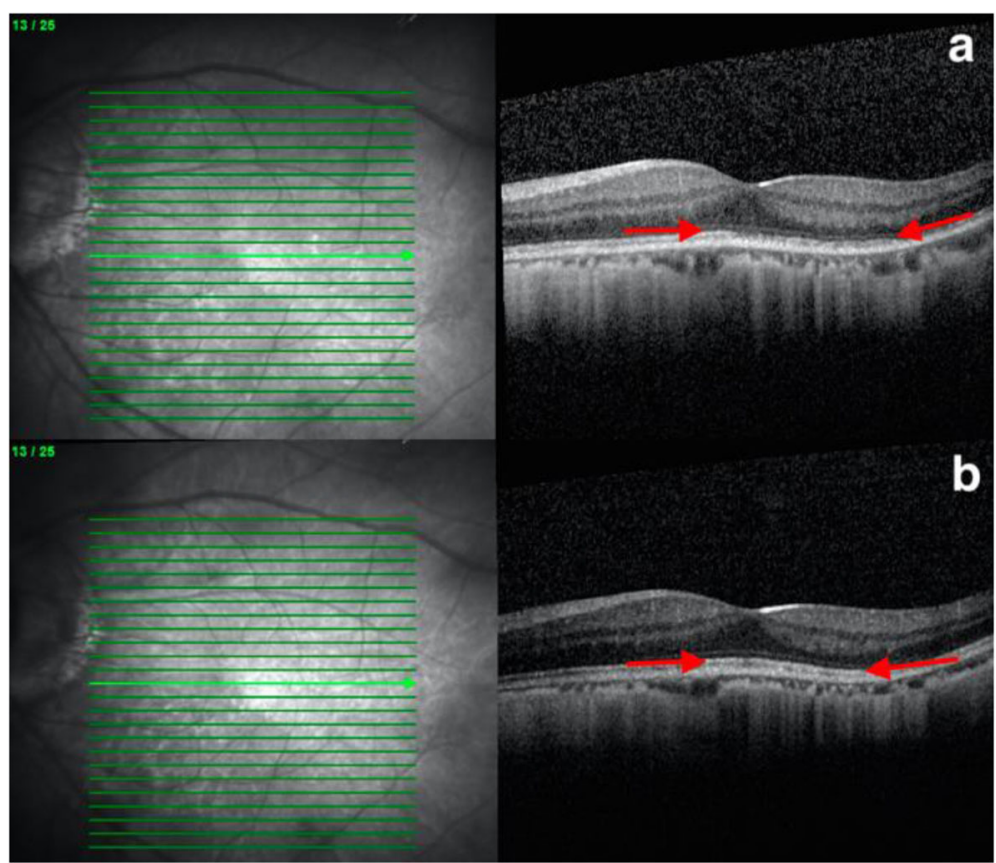

Fig. 3 OCT revealed change of IS/OS junction before and after treatment. a On the next day after onset of symptom, OCT revealed parafoveal loss (between arrows) of the photoreceptor IS/OS junction. b OCT revealed restoration (between arrows) of IS/OS junction after two weeks of IVI of Ozurdex

prednisolone monthly tapering with $30 \mathrm{mg} /$ day, $20 \mathrm{mg} /$ day, $15 \mathrm{mg} /$ day, $10 \mathrm{mg} /$ day, $5 \mathrm{mg} /$ day (each dose for one month). Their final logMAR BCVA $(0.16 \pm 0.52)$ was significantly better than the pretreatment value $(0.56 \pm 0.60)(P=0.007)$, and the mean $\mathrm{MD}$ at the final visit $(-9.82 \pm 10.74 \mathrm{~dB})$ was significantly higher than the initial value $(-13.66 \pm 10.73 \mathrm{~dB})$ $(P=0.02)$. Chen [12] gave three patients intravenous pulse steroid therapy (methylprednisolone $1000 \mathrm{mg} /$ day for 3 days) as the initial treatment followed by oral prednisolone with gradual tapering within 3 months, and prescribed another five patients oral prednisolone $1 \mathrm{mg} / \mathrm{kg} /$ day as the initial treatment with gradual tapering in 3 months. Although all of the patients had improvement of visual field eventually; however, recurrence was noted in two patients and three patients experienced deterioration of the visual field after stopping or tapering the systemic steroid. Immunotherapy with azathioprine [24] or mycophenolate [12] was added to patients with reactivation of the disease during prednisolone taper.

On the other hand, ocular penetration of systemically administered drugs could have been restricted by the blood-retinal barrier, and the diluting effect of blood volume requires larger systemic doses [26]. Ozurdex contained micronized dexamethasone $0.7 \mathrm{mg}$ in a biodegradable copolymer, and could be effective up to 6 months after intravitreal injection [26]. Hence, IVI of steroid might be beneficial for patients with AZOOR in the rationale.

Studies have demonstrated that visual functions in AZOOR patients improved spontaneously, [24] whereas systemic steroid treatment showed benefits to visual outcome as well [12, 24]. Meanwhile, improvement of visual field defects was correlated with choroidal thickness reduction, [27] possibly implying "inflammatory" pattern in the choroid in the acute stage [28]. Our AZOOR case is the first to show improved VA and recovery of the IS/OS junction after IVI of Ozurdex. During the 13-month followup, his condition remained stable. However, we cannot rule out the residual or delayed effects from the systemic steroid treatment received prior to the administration of Ozurdex or the possibility of spontaneous recovery. Therefore, the reliability of intravitreal injection treatment with sustained released steroid need further elucidation with future clinical research of AZOOR.

\section{Abbreviations}

AIR: Autoimmune retinopathy; AZOOR: Acute zonal occult outer retinopathy; COST: The cone outer segment tip; D: Diopter; ERG: Electroretinogram;

FA: Fluorescein Angiography; IOP: Intraocular pressure; IS/OS junction: Inner/ outer segment; IVI: Intravitreal injection; OCT: Optical coherence tomography; RPE: Retinal pigment epithelial; VA: Visual acuity; VF: Visual field 


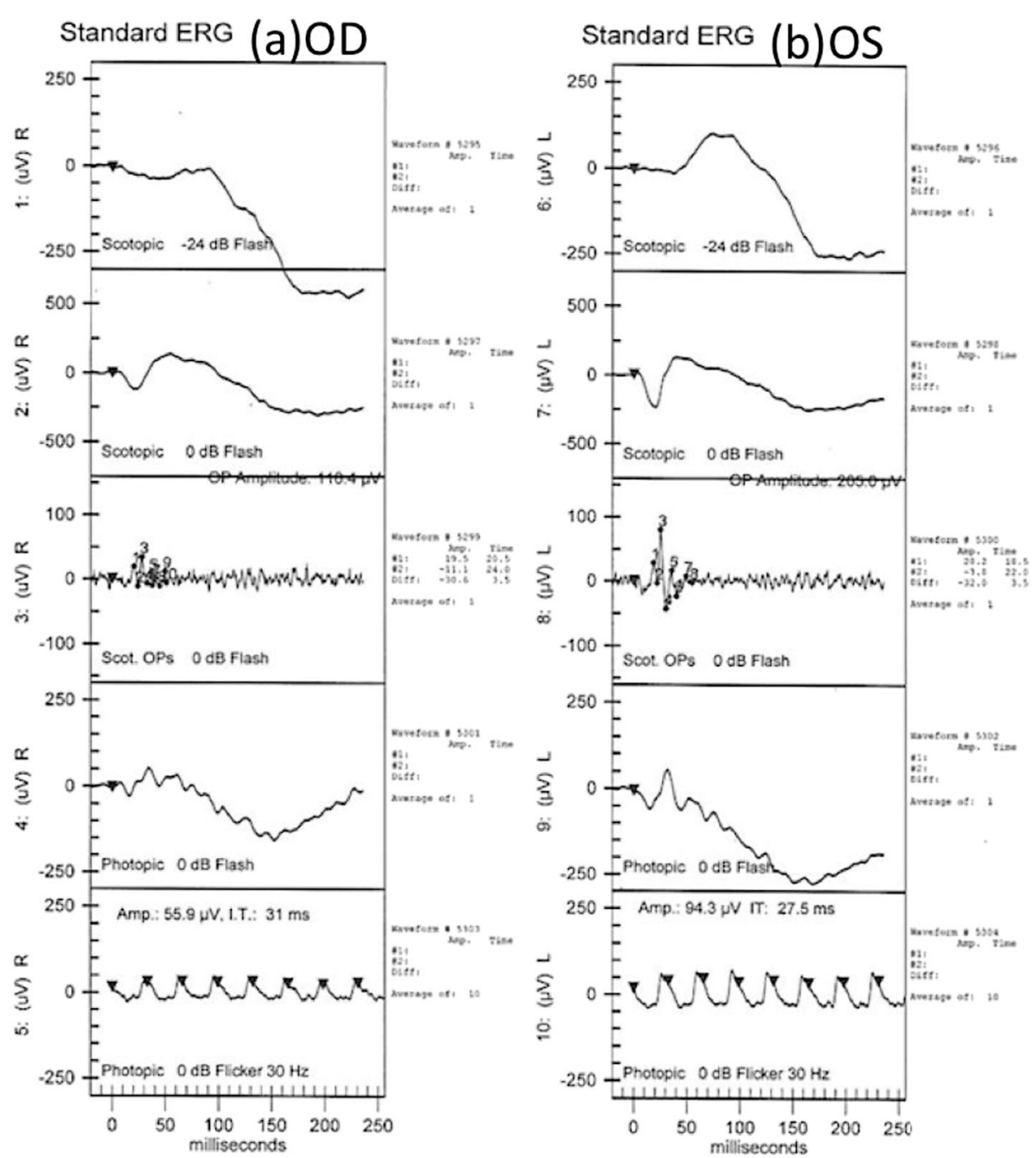

Fig. $4(\mathbf{a}, \mathbf{b})$ ERG illustrated decreased amplitude of b wave of scotopic stimulations in both eyes

Table 1 Relevant medical history and interventions

\begin{tabular}{|c|c|c|c|}
\hline Date & $\begin{array}{l}\text { Summaries from Initial } \\
\text { and Follow-up Visits }\end{array}$ & Diagnostic Testing & Interventions \\
\hline $2015 / 11 / 20$ & vision deteriorated(OS) & $\begin{array}{l}\text { visual acuity (VA)20/20 (OD) } \\
\text { and 20/32 (OS) }\end{array}$ & \\
\hline $2015 / 11 / 24$ & Still central scotoma (OS) & $\begin{array}{l}\text { FA: hyperfluorescence (OS) and } \\
\text { inflammation of retinal small } \\
\text { vessels. OCT: parafoveal loss of } \\
\text { the photoreceptor IS/OS junction }\end{array}$ & Dexamethasone $6 \mathrm{mg}$ daily \\
\hline 2015/12/02 & Still central scotoma (OS) & stationary & $\begin{array}{l}\text { Taper dexamethasone to } \\
4 \text { mg daily, and add } \\
\text { Amlodipine } 5 \text { mg daily }\end{array}$ \\
\hline 2015/12/15 & Still central scotoma (OS) & stationary & $\begin{array}{l}\text { Intravitreal injection of } \\
\text { Ozurdex. }\end{array}$ \\
\hline $2015 / 12 / 30$ & Vision improved (OS) & $\begin{array}{l}\text { VA (OS) improved to } 20 / 20 \\
\text { OCT: improvement of the } \\
\text { IS/OS junction disruption }\end{array}$ & \\
\hline 2017/01/18 & Vision stable(OS) & $\begin{array}{l}\text { VA (OS) was } 20 / 20 \\
\text { OCT: no sign of IS/OS junction } \\
\text { disruption. }\end{array}$ & \\
\hline
\end{tabular}




\section{Acknowledgements}

We acknowledge the contribution of Yao-Tseng Wen, PhD for the format of the reference.

\section{Funding}

The manuscript was not funded.

\section{Availability of data and materials}

All of the data supporting our findings are contained within the manuscript.

\section{Authors' contributions}

YCK drafted the manuscript, did the literature review, discussed the case with the other clinicians. Consent the patient for follow-up visits and publications. NC examined the patient, and critically analyzed the manuscript. RKT examined and managed the patient, offered valuable insight into the diagnosis and management of the patient, and critically revised the manuscript. He is the mentor of the authors. All authors read and approved the final manuscript.

\section{Ethics approval and consent to participate}

Not applicable.

\section{Consent for publication}

Our patient consent to publish the image of eyes and other results of his medical examination in this manuscript or paper which may be published in your journal. Written consent was obtained from our patient.

\section{Competing interests}

The authors declare that they have no competing interests.

\section{Publisher's Note}

Springer Nature remains neutral with regard to jurisdictional claims in published maps and institutional affiliations.

\section{Author details}

'Department of Ophthalmology, Buddhist Tzu Chi General Hospital, Hualien, Taiwan. ${ }^{2}$ Institute of Eye Research, Buddhist Tzu Chi General Hospital, Tzu Chi University, 707, Sec. 3, Chung-Yang Rd., Hualien 970, Taiwan. ${ }^{3}$ Institute of Medical Sciences, Tzu Chi University, Hualien, Taiwan.

Received: 19 August 2017 Accepted: 29 November 2017 Published online: 06 December 2017

\section{References}

1. Gass JD. Acute zonal occult outer retinopathy. Donders lecture: The Netherlands ophthalmological society, Maastricht, Holland, June 19, 1992. J Clin Neuroophthalmol. 1993;13(2):79-97.

2. Arai M, Nao-i N, Sawada A, Hayashida T. Multifocal electroretinogram indicates visual field loss in acute zonal occult outer retinopathy. Am J Ophthalmol. 1998;126(3):466-9.

3. Gass JD, Agarwal A, Scott IU. Acute zonal occult outer retinopathy: a longterm follow-up study. Am J Ophthalmol. 2002;134(3):329-39.

4. Francis PJ, Marinescu A, Fitzke FW, Bird AC, Holder GE. Acute zonal occult outer retinopathy: towards a set of diagnostic criteria. Br J Ophthalmol. 2005;89(1):70-3.

5. Spaide RF, Koizumi H, Freund KB. Photoreceptor outer segment abnormalities as a cause of blind spot enlargement in acute zonal occult outer retinopathy-complex diseases. Am J Ophthalmol. 2008;146(1):111-20.

6. Zibrandtsen N, Munch IC, Klemp K, Jorgensen TM, Sander B, Larsen M. Photoreceptor atrophy in acute zonal occult outer retinopathy. Acta Ophthalmol. 2008;86(8):913-6.

7. Monson DM, Smith JR. Acute zonal occult outer retinopathy. Surv Ophthalmol. 2011;56(1):23-35.

8. Mrejen S, Khan S, Gallego-Pinazo R, Jampol LM, Yannuzzi LA. Acute zonal occult outer retinopathy: a classification based on multimodal imaging. JAMA Ophthalmol. 2014;132(9):1089-98.

9. Srinivasan VJ, Monson BK, Wojtkowski M, Bilonick RA, Gorczynska I, Chen R, Duker JS, Schuman JS, Fujimoto JG. Characterization of outer retinal morphology with high-speed, ultrahigh-resolution optical coherence tomography. Invest Ophthalmol Vis Sci. 2008;49(4):1571-9.
10. So K, Shinoda K, Matsumoto CS, Satofuka S, Imamura Y, Mizota A. Focal functional and microstructural changes of photoreceptors in eyes with acute zonal occult outer retinopathy. Case Rep Ophthalmol. 2011;2(3):307-13.

11. Neutzner RV, Jager M, Friedburg C, Deeg CA, Lorenz B. Blind spot enlargement syndrome in acute zonal occult outer retinopathy with detection of autoantibodies against the retinal antigens CRALBP and S-ag. Ophthalmologe. 2011;108(11):1045-9.

12. Chen $\mathrm{SN}$, Yang $\mathrm{CH}$, Yang $\mathrm{CM}$. Systemic corticosteroids therapy in the management of acute zonal occult outer retinopathy. J Ophthalmol. 2015;2015:793026

13. Jampol LM, Wiredu A, MEWDS MFC, PIC AMN. AIBSE, and AZOOR: one disease or many? Retina. 1995;15(5):373-8.

14. Jampol LM, Becker KG. White spot syndromes of the retina: a hypothesis based on the common genetic hypothesis of autoimmune/inflammatory disease. Am J Ophthalmol. 2003:135(3):376-9.

15. Carrasco L, Ramos M, Galisteo R, Pisa D, Fresno M, Gonzalez ME. Isolation of Candida Famata from a patient with acute zonal occult outer retinopathy. J Clin Microbiol. 2005;43(2):635-40.

16. Pisa D, Ramos M, Garcia P, Escoto R, Barraquer R, Molina S, Carrasco L. Fungal infection in patients with serpiginous choroiditis or acute zonal occult outer retinopathy. J Clin Microbiol. 2008;46(1):130-5.

17. Mahendradas P, Shetty R, Avadhani K, Ross C, Gupta A, Shetty BK Polycythemia vera and increased hemophilic factor VIII causing acute zonal occult outer retinopathy: a case report. Ocul Immunol Inflamm. 2010;18(4):319-21.

18. Cheung MC, Nune GC, Hwang DG, Sutter EE, Duncan JL. Acute zonal occult outer retinopathy in a patient with graft-versus-host disease. Am J Ophthalmol. 2004;138(6):1058-60.

19. Mahajan VB, Stone EM. Patients with an acute zonal occult outer retinopathy-like illness rapidly improve with valacyclovir treatment. Am J Ophthalmol. 2010;150(4):511-8.

20. Tagami M, Matsumiya W, Imai H, Kusuhara S, Honda S, Azumi A. Autologous antibodies to outer retina in acute zonal occult outer retinopathy. Jpn J Ophthalmol. 2014;58(6):462-72.

21. Qian CX, Wang A, DeMill DL, Jayasundera T, Branham K, Abalem MF, Khan $\mathrm{N}$, Heckenlively JR. Prevalence of Antiretinal antibodies in acute zonal occult outer retinopathy: a comprehensive review of 25 cases. Am J Ophthalmol. 2017:176:210-8

22. Heckenlively JR, Ferreyra HA. Autoimmune retinopathy: a review and summary. Semin Immunopathol. 2008;30(2):127-34.

23. Kitakawa T, Hayashi T, Takashina H, Mitooka K, Gekka T, Tsuneoka H. Improvement of central visual function following steroid pulse therapy in acute zonal occult outer retinopathy. Doc Ophthalmol. 2012;124(3):249-54

24. Saito S, Saito W, Saito M, Hashimoto Y, Mori S, Noda K, Namba K, Ishida S. Acute zonal occult outer retinopathy in Japanese patients: clinical features, visual function, and factors affecting visual function. PLoS One. 2015;10(4):e0125133

25. Neri P, Ricci F, Giovannini A, Arapi I, De Felici C, Cusumano A, Mariotti C. Successful treatment of an overlapping choriocapillaritis between multifocal choroiditis and acute zonal occult outer retinopathy (AZOOR) with adalimumab (Humira). Int Ophthalmol. 2014;34(2):359-64.

26. Dugel PU, Bandello F, Loewenstein A. Dexamethasone intravitreal implant in the treatment of diabetic macular edema. Clin Ophthalmol. 2015:9:1321-35.

27. Hashimoto Y, Saito W, Saito M, Hasegawa Y, Takita A, Mori S, Noda K, Ishida S. Relationship between choroidal thickness and visual field impairment in acute zonal occult outer retinopathy. J Ophthalmol. 2017;2017:2371032.

28. Hirooka K, Saito W, Namba K, Takemoto Y, Mizuuchi K, Uno T, Tagawa Y, Hashimoto $Y$, Ishida S. Relationship between choroidal blood flow velocity and choroidal thickness during systemic corticosteroid therapy for Vogt-Koyanagi-Harada disease. Graefes Arch Clin Exp Ophthalmol. 2015:253(4):609-17. 